\title{
Endovascular treatment of a carotid pseudoaneurysm with covered stents: Case report and review of the literature.
}

\author{
Maen Aboul Hosn $^{1 *}$, Rachael Nicholson ${ }^{1}$, Timothy Kresowik ${ }^{1}$, William J Sharp ${ }^{1}$, Neelima Katragunta ${ }^{1}$, \\ Luigi Pascarella ${ }^{2}$ \\ ${ }^{1}$ Department of Vascular Surgery, University of Iowa Hospitals and Clinics, Iowa City, IA, USA \\ ${ }^{2}$ Department of Vascular Surgery, University of North Carolina, Chapel Hill, NC, USA
}

\begin{abstract}
Although endovascular placement of covered stent grafts has emerged as the primary treatment modality for arterial aneurysms, pseudoaneurysms and traumatic arterial injuries especially in high risk patients and in those with surgically inaccessible lesions, their use in carotid pseudoaneurysms remains uncommon and is limited to case reports and small case series. We report a successful endovascular treatment of a spontaneous right carotid pseudoaneurysm with a covered stent with no immediate perioperative complication and complete exclusion of the pseudoaneurysm on follow up imaging. We also review the literature on the treatment of carotid pseudoaneurysms with covered stents.
\end{abstract}

Keywords: Carotid, Pseudoaneurysm, Endovascular, Covered stent.

Accepted on November $5^{\text {th }}, 2017$

\section{Introduction}

Over the past years, the use of covered stent grafts has emerged as the primary treatment modality for arterial aneurysms, pseudoaneurysms and traumatic arterial injuries. This is especially true in high risk patients and in those with surgically inaccessible lesions as it offers a less morbid alternative. Extra cranial carotid pseudoaneurysms, in particular, are rare entities mostly described in the setting of trauma, infection, and head and neck carcinoma and have traditionally been managed with open surgical repair. Endovascular implantation of covered stent grafts for carotid pseudoaneurysms remains uncommon and is limited to case reports and small case series. We present a rare case of a spontaneous extra cranial carotid artery pseudoaneurysm which was treated with a covered stent graft placement in a patient with a recent history of an iliac pseudoaneurysm managed in a similar fashion. We also review the current literature on the use of covered stent grafts in extra cranial carotid pseudoaneurysms.

\section{Case Report}

A 66-year-old male with known hypertension, congestive heart failure, history of back melanoma excision, history of gastrointestinal stroma tumor status post partial gastrectomy and known recurrent bladder cancer involving the ureteral orifice for which he underwent resection and started on intravesical gemcitabine and docetaxel monthly. After receiving a total of 24 doses of his chemotherapy, a positron emission tomography scan (PET) performed at that point was negative for any distant metastasis. However, it revealed hypermetabolic multifocal vasculitis involving the proximal right internal carotid, distal abdominal aorta, left common iliac, and left internal iliac arteries (Figure 1). He continued to receive his intravesical chemotherapy with a repeat PET done 6 months later showing a hypermetabolic signal and possible aneurysmal dilatation of the left common iliac artery with surrounding inflammation. A computed tomography scan (CT) with contrast was then ordered and it showed a $4.9 \mathrm{~cm}$ left common iliac pseudoaneurysm at the level of the bifurcation. The patient was asymptomatic and subsequently underwent staged left hypogastric occlusion with an Amplatzer plug followed by an elective left common iliac artery stenting with a $13 \mathrm{~mm} \times 100 \mathrm{~mm}$ Viabahn stent (W.L. Gore, Flagstaff, AZ, USA). During follow up in clinic a month later, a pulsatile right neck mass was noticed on

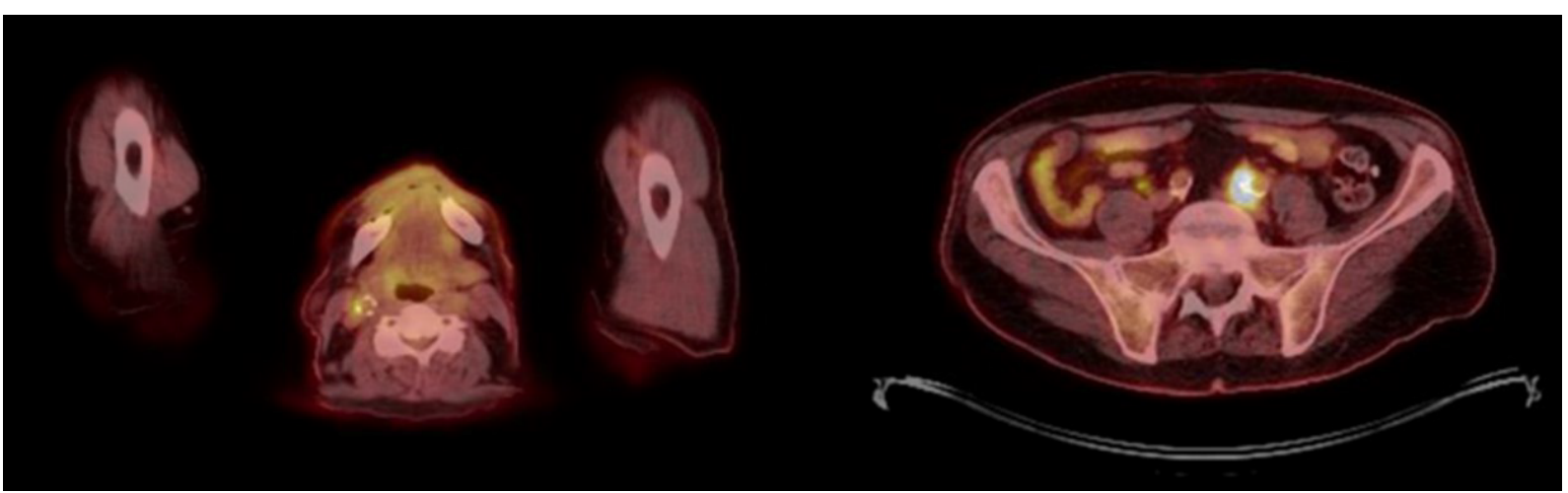

Figure 1. PET scan showing hypermetabolic multifocal vasculitis involving the proximal right internal carotid and the left common iliac arteries. 
Citation: Hosn MA, Nicholson R, Kresowik T, et al. Endovascular treatment of a carotid pseudoaneurysm with covered stents: Case report and review of the literature. Ann Cardiovasc Thorac Surg. 2017;1(1):11-14.

examination which the patient had reportedly noticed a few weeks earlier. Upon review of his most recent PET/CT, a right carotid pseudoaneurysm was identified. A neck CTA was then obtained revealing the pseudoaneurysm to be $3.3 \mathrm{~cm} \times 3.5 \mathrm{~cm}$ $\times 3.9 \mathrm{~cm}$ with approximately $10 \mathrm{~mm} \times 14 \mathrm{~mm}$ residual filling lumen (Figure 2) which was significantly larger compared to the PET/CT from two months prior. He was also complaining of pain in his neck but his inflammatory markers including white blood cell count, ESR and CRP were all within normal limits. Given his overall condition as well as the size of the pseudoaneurysm and surrounding inflammation, we opted to proceed with endovascular exclusion of the pseudoaneurysm with a covered stent. The procedure was performed under local anesthesia with selective right carotid angiography confirming the location of the pseudoaneurysm at the level of the carotid bifurcation with some extension into the right ICA. The patient became agitated during the procedure and the decision was made to proceed without and embolic protection device in order to expedite the procedure. The pseudoaneurysm was successfully covered with $8 \mathrm{~mm} \times$ $50 \mathrm{~mm}$ and $8 \mathrm{~mm} \times 25 \mathrm{~mm}$ overlapping Viabahn covered stents (W.L. Gore, Flagstaff, AZ, USA) with no immediate complications. Completion angiography showed adequate exclusion of the pseudoaneurysm. Although there was a small type II endoleak from retrograde flow from the external carotid artery, it did not appear to be filling the pseudoaneurysm and no further interventions were performed (Figure 3). A total of 7000 units of heparin were administered during the procedure. Preoperatively, the patient did not exhibit any neurological deficits and a carotid duplex ultrasound showed complete exclusion of the pseudoaneurysm with no flow seen in the sac (Figure 4). He was discharged home the following day on daily aspirin $(81 \mathrm{mg})$ and a repeat CT angiography done at 6 months revealed a patent ICA with complete resolution of the hematoma and no endoleaks.

\section{Review of Literature}

We used the PubMed and OVID Medline databases in order to conduct a systematic review of the English-speaking medical literature and identify all publications documenting the use of covered stents in extracranial carotid artery pseudoaneurysms. Keywords such as 'Stents' [Mesh] OR 'Covered stents' [Mesh] AND 'Carotid Artery Pseudoaneurysm'[Mesh] NOT 'Intracranial Arterial Diseases' [Mesh] NOT 'Carotid-Cavernous Sinus Fistula'[Mesh] NOT Carotid Artery aneurysm'[Mesh] were used. We decided to include only case series and reviews with at least 5 cases with documented follow up and as such our initial search results were further filtered. Our final yield was four publications that fulfilled these criteria (Table 1) [1-4].

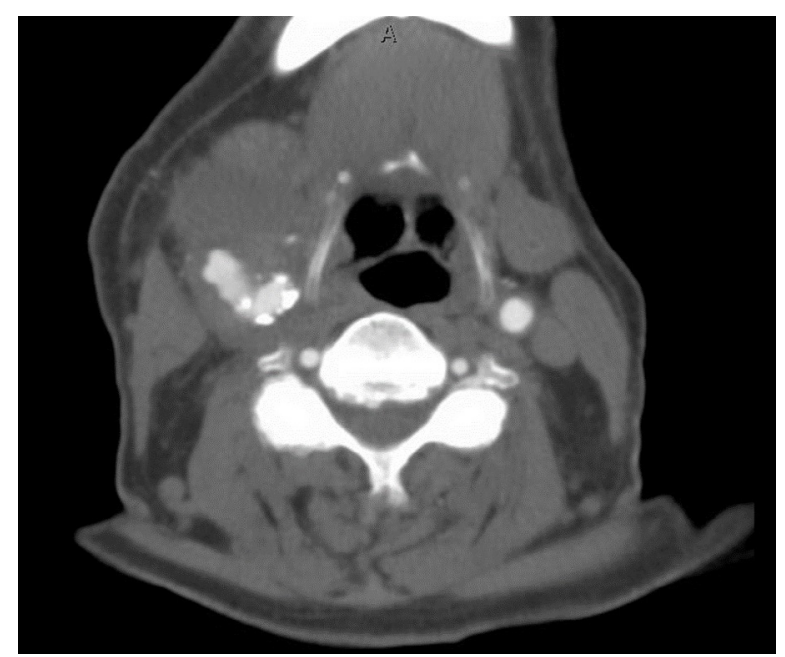

Figure 2. CTA of the neck showing the right carotid pseudoaneurysm.

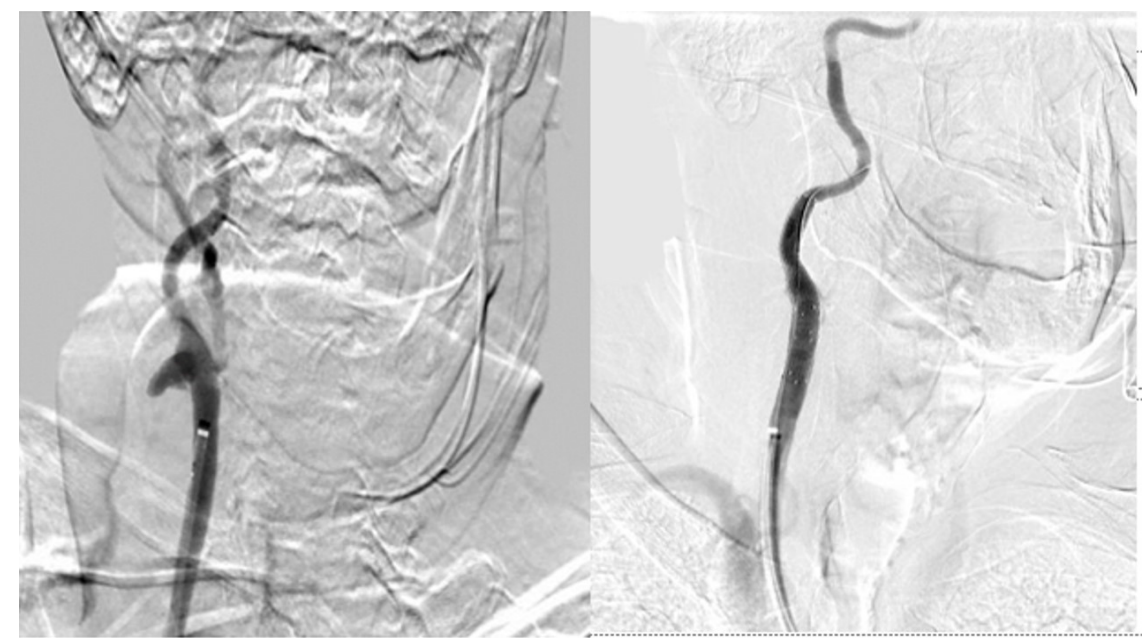

Figure 3. Selective right carotid angiography before and after exclusion of the pseudo aneurysm. 


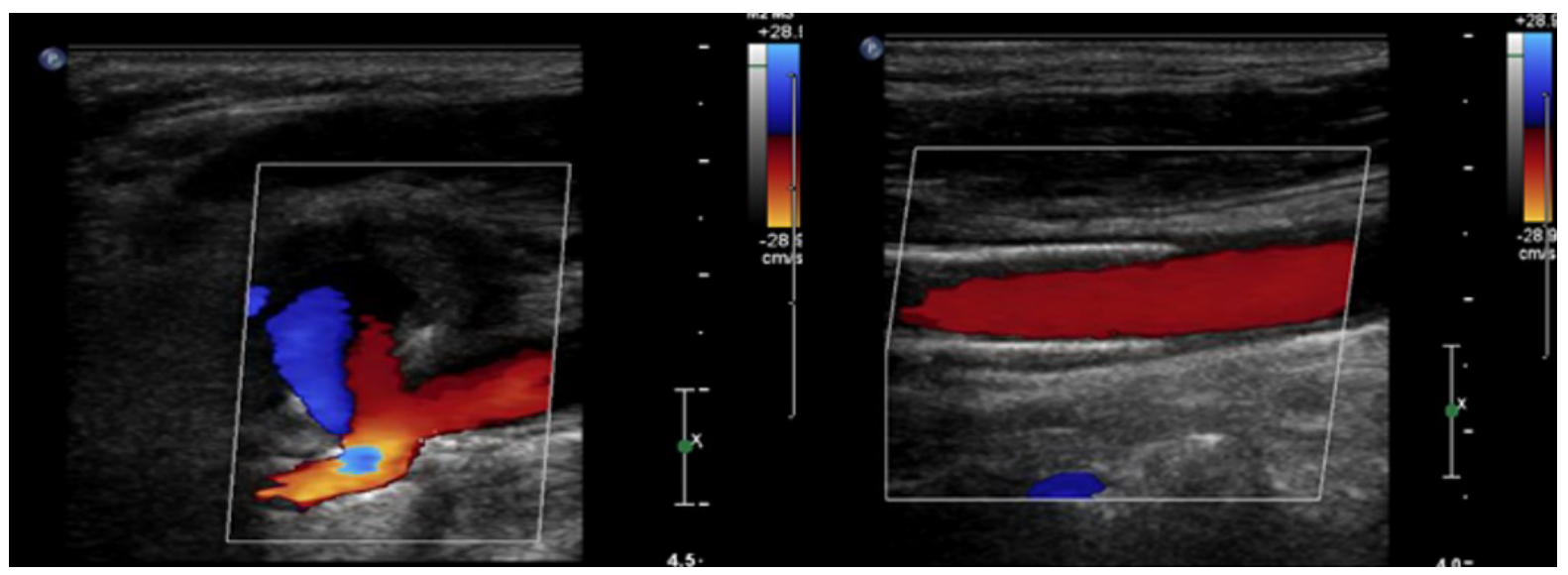

Figure 4. Duplex ultrasound showing a right common carotid pseudoaneurysm before repair (left) and complete exclusion of the pseudoaneurysm after stenting (right).

Table 1. List of carotid pseudoaneurysm cases treated with covered stents.

\begin{tabular}{|c|c|c|c|c|c|}
\hline Author & Year & $\begin{array}{c}\text { Number of patients with } \\
\text { carotid PSA }\end{array}$ & Stents used & $\begin{array}{c}\text { Technical } \\
\text { success }\end{array}$ & Follow up \\
\hline Saatci & 2004 & 24 & Jostent (24) & $96 \%$ & Nome \\
\hline Yi & 2008 & 9 & Fluency (6), Jostent (2), Viabahn (1), & iCast (1) & Distal embolization (1) \\
\hline Hoppe & 2008 & 9 & Viabahn (5), Fluency (4) & $100 \%$ & ICA dissection (1) \\
\hline Pulli & 2013 & 5 & Viabahn (3), Advanta (2) & 100\% & Type 1 endoleak \\
\hline
\end{tabular}

\section{Discussion}

Arterial pseudoaneurysms usually occur secondary to disruption of the arterial wall in which a hematoma forms around the vessel and subsequently dissolves leaving a cavity that communicates with the arterial lumen. The arterial pressure ultimately causes this cavity to enlarge forming an aneurysmal sac [5]. The natural history of pseudoaneurysms depends on their anatomic location and underlying etiology and can range from spontaneous thrombosis to continuous enlargement and rupture. Pseudoaneurysms of the extra cranial carotid artery are rare and have been mostly described in the setting of blunt and penetrating trauma. Other reported etiologies include infection, head and neck malignancies, radiation and iatrogenic injuries during procedures [6]. Such pseudoaneurysms rarely thrombose spontaneously owing to the high arterial flow as compared to other anatomical regions. Rupture and hemorrhage are also unusual. The majority of complications are neurologic in nature either due to embolic events or extrinsic nerve compression [7]. Other clinical presentations include a pulsatile cervical mass or bruit on physical examination. Our patient's carotid pseudoaneurysm had increased in size significantly since he was initially treated for his iliac pseudoaneurysm one month earlier and was now pulsatile on examination with the patient reporting increasing neck pain and dysphagia. The conventional treatment of carotid pseudoaneurysms has been primarily open surgical repair. However, these surgeries are technically challenging especially in those located near the skull base. They are also associated increased mortality and morbidity mainly due to the extensive exposure required [8]. In particular, excision of large carotid pseudoaneurysms carries a high risk of cranial nerve injury including the vagus, spinal accessory, hypoglossal and glossopharyngeal nerves with transient cranial nerve dysfunction reported in up to $20 \%$ of patients [9]. Endovascular repair of carotid pseudoaneurysms has recently emerged as an alternative treatment modality mainly due to advances in delivery systems and the increased experience with carotid stenting for carotid occlusive disease. Available endovascular options include bare stent placement with or without coil embolization, coil embolization alone or, more recently, covered stent grafts. The data on the use of covered stent grafts in the treatment of carotid pseudoaneurysms is limited to small case series but is nonetheless promising. Saatci et al reported the use of covered stents in 25 patients with internal carotid artery (ICA) pseudoaneurysms, the majority of which were posttraumatic in nature. Immediate exclusion of the pseudoaneurysm was seen in 23 patients and the remaining two had an endoleak, one of which resolved spontaneously and the other required an additional placement of a bare-metal stent. No peri or postoperative mortalities or morbidities were reported with midterm follow up in 21 patients revealing patency of the ICA with continued aneurysm exclusion [1]. Yi et al. published their experience with endovascular treatment of carotid and vertebral pseudoaneurysms using covered stent grafts in 10 patients. In their series, successful pseudoaneurysm exclusion was seen in all patients with one distal embolic event reported. Also, one stent occlusion was seen on follow up 4.5 months later in a patient treated for a vertebral artery pseudoaneurysm who was not on antiplatelet therapy [2]. A retrospective study on the use of carotid stent grafts in an emergent setting was also published by Hoppe et al. whose series included 25 patients, 13 of which had carotid blow-out syndrome and 11 had carotid pseudoaneurysms. The overall patient mortality rate was $36 \%$, all with carotid blow-out syndrome. At 6-month angiographic follow-up, widely patent stent-grafts were found in all patients treated for pseudoaneurysms and three of the remaining patients with 
carotid blow-out syndrome [3]. Although the preliminary and midterm results are promising, the lack of data on long term results following carotid pseudoaneurysm stenting with covered stent grafts remains a major concern. Recently, Pulli et al. published their two year follow up data on 5 patients who underwent endovascular interventions for carotid pseudoaneurysms, four of which were managed with covered stent grafts. At 24 months, all patients were symptom free with patent ICA and no pseudoaneurysm recurrence [4]. In our case, there was persistent filling of the pseudoaneurysm sac after deployment of the first Viabahn stent-graft suggestive of a type 1 endoleak. This was managed by deployment of a second Viabahn stent-graft combined with balloon angioplasty with completion angiography revealing complete exclusion of the pseudoaneurysm. Although there was an immediate small type 2 endoleak with retrograde filling from the external carotid artery, this had resolved on follow up carotid duplex on the first post-operative day [10].

\section{Conclusion}

Although the use of covered stents to treat carotid pseudoaneurysms remains uncommon with limited literature to validate its use, our case report adds to the small but growing number of reports on the technical feasibility and safety of such procedure in specific clinical settings. This report also highlights the unusual case of multifocal vasculitis and inflammation documented by PET scan progressing in pseudoaneurysm formation although the actual pathology remains unclear since no tissue samples have been obtained for analysis.

\section{References}

1. Saatci I, Cekirge HS, Ozturk MH, et al. Treatment of internal carotid artery aneurysms with a covered stent: Experience in 24 patients with mid-term follow-up results. Am J Neuroradiol. 2004;25(10):1742-9.
2. Yi AC, Palmer E, Luh GY, et al. Endovascular treatment of carotid and vertebral pseudoaneurysms with covered stents. Am J Neuroradiol. 2008;29(5):983-7.

3. Hoppe H, Barnwell SL, Nesbit GM, et al. Stent-grafts in the treatment of emergent or urgent carotid artery disease: Review of 25 cases. J Vasc Interv Radiol. 2008;19(1):31-41.

4. Pulli R, Dorigo W, Pratesi G, et al. Single-center experience on endovascular repair of noninfected extracranial internal carotid artery pseudoaneurysms. Ann Vasc Surg. 2013;27(5):13-7.

5. Beale PJ. Late development of a false aneurysm of the common carotid artery. Br J Surg. 1971;58:76-8.

6. Alaraj A, Wallace A, Amin-Hanjani S, et al. Endovascular implantation of covered stents in the extracranial carotid and vertebral arteries: Case series and review of the literature. Surg Neurol Int. 2011;2:67.

7. Srivastava SD, Eagleton MJ, O'Hara P, et al. Surgical repair of carotid artery aneurysms: A 10-year, single-center experience. Ann Vasc Surg. 2010;24(1):100-5.

8. Koenigsberg RA, Urrutia V, McCormick D, et al. Endovascular treatment of a left carotid artery "bowtie" pseudoaneurysm with a covered Wallgraft stent. J Neuroimaging. 2003;13(4):362-6.

9. El-Sabrout R, Cooley DA. Extracranial carotid artery aneurysms: Texas Heart Institute experience. J Vasc Surg. 2000;31(4):702-12.

10. Hosn MA. Endovascular treatment of a carotid pseudoaneurysm with covered stents: Case report and review of the literature. Poster session presented at: Society for Clinical Vascular Surgery annual meeting; 2016 March 12-16. http://www.epostersonline.com/scvs2016/node/500.

\section{*Correspondence to:}

Maen Aboul Hosn

Department of Vascular Surgery

University of Iowa Hospitals and Clinics

Iowa City

IA

USA

Tel: +1 (319) 936-3081

E-mail: maen-aboulhosn@uiowa.edu 